Open Access

\title{
$\beta 2$-adrenoceptor signaling regulates invadopodia formation to enhance tumor cell invasion
}

Sarah J. Creed ${ }^{1}$, Caroline P. Le', Mona Hassan' ${ }^{1}$, Cindy K. Pon' ${ }^{1}$, Sabine Albold ${ }^{1}$, Keefe T. Chan ${ }^{2,7}$, Matthew E. Berginski ${ }^{3}$, Zhendong Huang ${ }^{4}$, James E. Bear ${ }^{2}$, J. Robert Lane ${ }^{1}$, Michelle L. Halls ${ }^{1}$, Davide Ferrari ${ }^{4}$, Cameron J. Nowell ${ }^{1}$ and Erica K. Sloan ${ }^{1,5,6^{*}}$

\begin{abstract}
Introduction: For efficient metastatic dissemination, tumor cells form invadopodia to degrade and move through three-dimensional extracellular matrix. However, little is known about the conditions that favor invadopodia formation. Here, we investigated the effect of $\beta$-adrenoceptor signaling - which allows cells to respond to stress neurotransmitters - on the formation of invadopodia and examined the effect on tumor cell invasion.

Methods: To characterize the molecular and cellular mechanisms of $\beta$-adrenergic signaling on the invasive properties of breast cancer cells, we used functional cellular assays to quantify invadopodia formation and to evaluate cell invasion in two-dimensional and three-dimensional environments. The functional significance of $\beta$-adrenergic regulation of invadopodia was investigated in an orthotopic mouse model of spontaneous breast cancer metastasis.

Results: $\beta$-adrenoceptor activation increased the frequency of invadopodia-positive tumor cells and the number of invadopodia per cell. The effects were selectively mediated by the $\beta_{2}$-adrenoceptor subtype, which signaled through the canonical Src pathway to regulate invadopodia formation. Increased invadopodia occurred at the expense of focal adhesion formation, resulting in a switch to increased tumor cell invasion through three-dimensional extracellular matrix. $\beta_{2}$-adrenoceptor signaling increased invasion of tumor cells from explanted primary tumors through surrounding extracellular matrix, suggesting a possible mechanism for the observed increased spontaneous tumor cell dissemination in vivo. Selective antagonism of $\beta_{2}$-adrenoceptors blocked invadopodia formation, suggesting a pharmacological strategy to prevent tumor cell dissemination.

Conclusion: These findings provide insight into conditions that control tumor cell invasion by identifying signaling through $\beta_{2}$-adrenoceptors as a regulator of invadopodia formation. These findings suggest novel pharmacological strategies for intervention, by using $\beta$-blockers to target $\beta_{2}$-adrenoceptors to limit tumor cell dissemination and metastasis.
\end{abstract}

\footnotetext{
* Correspondence: erica.sloan@monash.edu

${ }^{1}$ Drug Discovery Biology Theme, Monash Institute of Pharmaceutical

Sciences, Monash University, Parkville, VIC 3052, Australia

${ }^{5}$ Cousins Center for PNI, UCLA Semel Institute, and Jonsson Comprehensive

Cancer Center, University of California Los Angeles, Los Angeles, CA 90095,

USA

Full list of author information is available at the end of the article
}

\section{Biomed Central}

(C) 2015 Creed et al. Open Access This article is distributed under the terms of the Creative Commons Attribution 4.0 International License (http://creativecommons.org/licenses/by/4.0/), which permits unrestricted use, distribution, and reproduction in any medium, provided you give appropriate credit to the original author(s) and the source, provide a link to the Creative Commons license, and indicate if changes were made. The Creative Commons Public Domain Dedication waiver (http://creativecommons.org/publicdomain/zero/1.0/) applies to the data made available in this article, unless otherwise stated. 


\section{Introduction}

Metastasis is the main cause of death from cancer, and involves the dissemination of cancer cells from the primary tumor to colonize distant tissues [1]. To aid dissemination, cancer cells form specialized actin-rich structures called invadopodia that facilitate invasion through the basement membrane and surrounding stroma [2]. Invadopodia produce and localize matrix metalloproteases (MMPs) to focally degrade surrounding extracellular matrix. Src has been shown to play a key role in invadopodia formation and invasion [3, 4]. Src may be recruited away from focal adhesions $[5,6]$, resulting in a shift to invadopodia-mediated invasion, which favors tumor cell movement through the surrounding three-dimensional extracellular matrix and leads to tumor cell dissemination to distant organs.

Tumor progression and metastasis are regulated by bidirectional signaling between tumor cells and their surrounding microenvironment [7]. $\beta$-adrenoceptors ( $\beta$ ARs) are found on both tumor cells and untransformed cells in the tumor microenvironment, and allow for cellular response to neural signals $[8,9]$. Neurotransmitters including noradrenaline and adrenaline are released during stress and bind to $\beta A R s$ [10]. Activation of $\beta A R$ induces intracellular signaling cascades that accumulate cAMP, activate PKA, and regulate gene transcription to modify cell behavior [11]. $\beta$ AR signaling drives metastasis [12-14], and Src activation has been implicated in $\beta A R$ regulation of metastasis [15]. However, the cellular mechanisms by which $\beta$ ARs drive tumor cell dissemination remain elusive.

To investigate, we explored the effect of $\beta A R$ signaling on the molecular and cellular mechanisms of tumor cell invasion. Using functional assays we found that $\beta A R$ signaling enhanced invadopodia formation through canonical Src signaling pathways. Increased invadopodia formation was associated with loss of focal adhesions and enhanced tumor cell invasion through three-dimensional extracellular matrix. These effects were selectively mediated by the $\beta_{2}$ AR subtype, which enhanced tumor cell invasion from primary mammary tumors and increased metastasis in vivo. $\beta_{2} \mathrm{AR}$ regulation of invadopodia could be reversed by pharmacological blockade, suggesting a strategy to reduce tumor cell dissemination in breast cancer.

\section{Methods}

Reagents

CGP-20712A dihydrochloride, ICI-118551 hydrochloride, and xamoterol hemifumerate were sourced from Tocris Bioscience (Bristol, UK); formoterol fumarate from BioNet (Cornwall, UK); and PP2 Src inhibitor from Calbiochem (Alexandria, Australia). Agonists were used at $0.5 \mu \mathrm{M}$ and antagonists at $0.05 \mu \mathrm{M}$ unless otherwise stated. Other reagents and chemicals were from Sigma-Aldrich (Castle Hill, Australia) unless otherwise stated.

\section{Cell culture and transduction}

The highly metastatic HM variant of MDA-MB-231 breast cancer cell line (described throughout as MDAMB-231) was a kind gift from Dr Zhou Ou, Fudan University Shanghai Cancer Center, China [16, 17]. The cell line identity was verified by karyotyping (CellBank Australia, Westmead, NSW Australia) and transduced to express codon-optimized luciferase 2, luc2 [13]. Cells were cultured in Dulbecco's modified Eagle's medium (DMEM; Invitrogen) supplemented with $10 \%$ fetal bovine serum (FBS). Cells were maintained at $37{ }^{\circ} \mathrm{C}$, in a humidified environment with $5 \% \mathrm{CO}_{2}$. The 66cl4 mouse mammary adenocarcinoma cell line (a kind gift from Prof Robin Anderson, Peter MacCallum Cancer Centre, East Melbourne, VIC, Australia) was cultured in $\alpha$-minimum essential medium (Invitrogen, Scoresby, VIC) containing $10 \%$ FBS [12]. These cell lines are characteristic of triplenegative breast cancer [18]. pLL5.0-LifeAct-GFP-2Aluc2 was generated by ligation of a sequence encoding the $2 \mathrm{~A}$ cleavage peptide and $l u c 2$ immediately $3^{\prime}$ of green fluorescent protein (GFP) in pLL5.0-LifeActGFP [19]. The luc2 sequence was PCR amplified from pGL4.10 (Promega, Madison, WI USA) using a modified $5^{\prime}$ primer that encoded the $2 \mathrm{~A}$ peptide sequence [20]. The product was sequence validated. Lentiviral production was performed as described previously [21] and a fluorescent population identified by fluorescence-activated cell sorting.

\section{Gene expression}

RNA was extracted using the RNeasy kit (Qiagen Chadstone, VIC Australia) and gene expression was quantified by quantitative RT-PCR using the iScript One-Step RT-PCR kit (Bio-Rad, Gladesville, NSW Australia) and Taqman probes (ADRB1, Hs02330048_s1; Adrb1, Mm00431701_s1; ADRB2, Hs00240532_s1; Adrb2, Mm02524224_s1; ADRB3, Hs00609046_m1; Adrb3, Mm02601819_g1; Life Technologies, Tullamarine, VIC Australia) and run on a CFX96 Real Time System (Bio-Rad). Triplicate determinations were evaluated by threshold cycle analysis and expressed relative to the housekeeping gene (ACTB, Hs99999903_m1; Actb, Mm00607939).

\section{CAMP assay}

cAMP accumulation was quantified by the Alphascreen cAMP kit (Perkin Elmer, Melbourne, VIC Australia) as per the manufacturer's instructions. Cells were serum-starved overnight and treated with antagonists in stimulation buffer for 30 minutes before addition of agonists for 10 minutes at $37{ }^{\circ} \mathrm{C}$. Cells were lysed in ice-cold ethanol, which was evaporated before reconstitution in detection buffer for cAMP assay. 


\section{Fixed invadopodia assay}

MDA-MB-231 cells expressing LifeAct-GFP-Luc2 were serum-starved overnight. Cells were preincubated with antagonists for 20 minutes before plating onto coverslips coated with Alexa Fluor-tagged gelatin or fibronectin in media containing $10 \%$ serum, \pm agonists or antagonists for 5 hours. Cells were fixed in $4 \%$ paraformaldehyde and nuclei counterstained with $1 \mu \mathrm{g} / \mathrm{ml}$ Hoechst 33242 . Cells were imaged on an SP8 confocal microscope (Leica Microsystems, North Ryde, NSW Australia) using a 63x PL APO CS2 1.4NA objective with excitation at $405 \mathrm{~nm}$, $488 \mathrm{~nm}, 561 \mathrm{~nm}$, and $633 \mathrm{~nm}$ and emission detectors set as follows: 415-485 PMT, 495-535 HyD, 570-635 PMT, and 645-705 PMT. Images were captured using LAS AF software version 3.2 (Leica Microsystems). Invadopodia were defined as actin-positive puncta overlying degraded (fluorescence-negative) matrix. The frequency of invadopodia-positive cells was manually counted in eight random fields of view from three independent experiments. $N \geq 80$ cells per treatment were quantified for each experiment. To determine the number of invadopodia per cell, image stacks were prepared and submitted to the Invadopodia Analysis Server [19], which uses a high-pass filter and threshold to identify regions of high actin concentration that colocalize with matrix degradation to identify active invadopodia. Average matrix fluorescence outside the cell bodies was set to 500 arbitrary units to allow the local difference values to be compared between extracellular matrix preparations [19]. Images were not preprocessed prior to submission to the Server. $N$ $\geq 130$ cells per treatment were quantified for each experiment. Experiments were conducted in triplicate.

\section{Immunofluorescence}

Focal adhesions were localized in MDA-MB-231 cells following fixation and permeabilization by incubating with $10 \mu \mathrm{g} / \mathrm{ml}$ anti-paxillin antibody (clone 5H11; Millipore, Bayswater, VIC Australia), followed by $1 \mu \mathrm{g} /$ $\mathrm{ml}$ Alexa Fluor 488-conjugated secondary antibody and $200 \mathrm{U} / \mathrm{ml}$ Alexa Fluor-647 phalloidin (Invitrogen) to costain actin. Nuclei were counterstained with $1 \mu \mathrm{g} / \mathrm{ml}$ Hoechst 33242. Cells were imaged using an SP8 confocal microscope as already described. Focal adhesions were measured in Image software (National Institutes of Health, Bethesda, MD USA) by drawing a region of interest across the longest axis of each paxillin-positive focal adhesion at the cell perimeter. Only adhesions greater than 3 pixels long were counted. Focal adhesions were analyzed from four random fields of view from each of two independent experiments. No nonlinear adjustments were made during image processing and all adjustments were applied to the entire image. Images were cropped to a single cell for presentation in figures without removing any additional image information. $\beta_{2} \mathrm{AR}$ was localized by immunostaining as described previously [12] in de-identified human breast cancer tissue obtained under approval from the Institutional Review Board. Samples were counterstained with antimacrophage antibody $(0.7 \mu \mathrm{g} / \mathrm{ml}$ Ham56; Dako, North Sydney, NSW Australia) to distinguish tumor cells from stromal cells.

\section{Two-dimensional migration assay}

Serum-starved LifeAct-GFP ${ }^{+}$MDA-MB-231 cells were seeded on fibronectin-coated chamber slides and treated \pm agonists/antagonists. Cells were imaged on a Ti-Ex microscope (Nikon Instruments, Melville, NY USA) using a 20× Plan Apo 0.6NA objective with fluorescence filters GFP excitation $470 / 40$ and emission 525/50, red fluorescence excitation $545 / 30$ and emission $620 / 60$, and far red excitation $620 / 60$ and emission 700/75. The microscope was fitted with an incubation chamber heated to $37{ }^{\circ} \mathrm{C}$ and humidified $\mathrm{CO}_{2}$ was supplied to the cells. Images were captured every 20 minutes for 16 hours using a SPOT Pursuit camera (Diagnostic Instruments, Victoria Park, WA Australia) and MetaMorph 7.8.0 software (Molecular Devices, Sunnyvale, CA USA). Time-series images were reconstructed and cells tracked manually using ImageJ software. No nonlinear adjustments were made during image processing and all adjustments were applied to the entire image. The experiment was conducted in duplicate.

\section{Single-cell three-dimensional migration assay}

Serum-starved LifeAct-GFP ${ }^{+}$MDA-MB-231 cells were resuspended in $1 \mathrm{mg} / \mathrm{ml}$ collagen (type I rat tail; MerkMillipore) \pm agonists/antagonists and the matrix was set at $37{ }^{\circ} \mathrm{C}$ for 20 minutes. Cells were imaged every $20 \mathrm{mi}-$ nutes for 16 hours using an A1R confocal microscope (Nikon) using a 20× Plan Apo 0.6NA objective with a correction collar, with excitation $488 \mathrm{~nm}$ and an emission 505-545 BP filter. The microscope was fitted with an incubation chamber heated to $37^{\circ} \mathrm{C}$ and humidified $\mathrm{CO}_{2}$ was supplied to cells. Z-stacks of $100 \mu \mathrm{m}$ in $20 \mu \mathrm{m}$ steps were captured using NIS Elements software (version 3.22; Nikon Instruments). Three-dimensional images were reconstructed in Imaris 7.6.4 software (Bitplane, Zurich Switzerland) and cell migration was tracked. Protrusionpositive cells were counted manually 6 hours after seeding. Any cell with a protrusion longer than half the cell body diameter was considered protrusion-positive. Images were prepared as maximum intensity projections. No nonlinear adjustments were made during image processing and all adjustments were applied to the entire image. Images were cropped to a single cell for presentation in figures without removing any additional image information. $N \geq 20$ cells were quantified per treatment. Experiments were conducted in triplicate. 


\section{Metastasis models and explants}

All procedures involving mice were carried out under protocols approved by the Institutional Animal Ethics Committee and in accordance with National Health and Medical Research Council animal ethics guidelines. MDA-MB-231 breast cancer cells were injected into the left fourth mammary fat pad of BALB/c nude mice as described previously [12]. Formoterol $(5 \mathrm{mg} / \mathrm{kg} /$ day $)$ or saline placebo was injected once daily by subcutaneous injection. Bioluminescence imaging was used to track tumor growth and metastatic progression using an IVIS Lumina II (Perkin Elmer) as described previously [12, 17]. Metastasis was confirmed by ex vivo imaging and hematoxylin and eosin staining. For analysis of tumor cell invasion from explanted primary tumors, a subset of mice were anesthetized on day 9 of mammary tumor growth and euthanized. Tumors were surgically removed from the mammary fat pad and embedded in $1 \mathrm{mg} / \mathrm{ml}$ collagen $\pm 0.5 \mu \mathrm{M}$ formoterol. Explants were maintained at $37{ }^{\circ} \mathrm{C}$ with $5 \% \mathrm{CO}_{2}$ and imaged every 2 days over a period of 8 days using an A1R confocal microscope (Nikon) as already described. Z-stacks were captured over $250 \mu \mathrm{m}$ at $50 \mu \mathrm{m}$ steps with large image format stitching of a $7 \times 7$ field of view grid. Time series images were reconstructed and analyzed using the Fiji distribution of ImageJ with StackReg plugin. Tumor boundaries were defined on the image that was acquired on day 0 and the area of tumor cell invasion of surrounding extracellular matrix outside this region of interest was then determined at subsequent time points. Five tumors were quantified per treatment. Two experimental replicates were conducted.

\section{Statistical analyses}

Generalized linear models were used to assess the relationship between treatments and experimental response [22]. The number of invadopodia per cell and the frequency of invadopodia-positive cells were modeled by binomial (logit link) and Poisson (log link) generalized linear models, respectively. Treatment differences for single continuous responses such as cell displacement and cAMP accumulation were analyzed by analysis of variance. For statistical analysis of in vivo metastatic progression and ex vivo invasion, the impact of $\beta_{2} \mathrm{AR}$ stimulation over time was analyzed using the linear model:

$$
\ln (Y)=\beta 0+\beta 1 t+\beta 2 I\{\text { formoterol }\} x t+\varepsilon
$$

where $Y$ is the luciferase activity (total flux, Fig. 5b) or the area of tumor cell invasion outside the tumor boundaries (Fig. 5c), $\beta 0$ is the intercept parameter, $t$ is time, $I$ \{formoterol\} (taking values 0 or 1 ) indicates the presence of formoterol, and $\varepsilon$ is a Gaussian random error. For the fitted models, we report estimated regression coefficients quantifying the relationship between the response and treatments, and the corresponding $p$ values for testing the null hypothesis of no treatment effect. For experiments involving multiple treatments, we tested simultaneous differences between pairs of treatment effects (Tukey's all-pair comparisons) [23]. Confidence intervals and $p$ values were adjusted at the nominal $5 \%$ significance level for multiple testing. Statistical analysis was carried out in the $\mathrm{R}$ computing environment [24]. Generalized linear models were fitted using the routine glm while multiple comparisons used the routine glht in the package multicomp [25].

\section{Results and Discussion}

We first confirmed expression of $\beta A R$ in MDA-MB-231 and 66cl4 tumor cells, which are breast cancer cells lines that respond to stress signaling with increased metastasis from primary orthotopic mammary tumors $[12,13]$. Expression analyses found higher $\beta_{2} A R$ than $\beta_{1}$ AR transcription in MDA-MB-231 cells, while $66 \mathrm{cl} 4$ expressed only $\beta_{2} A R$ (Fig. 1a). $\beta_{3} A R$ was not detectable in either cell line, although priming from these probes has been confirmed [26, 27]. Immunostaining confirmed that tumor cells from an archival clinical sample expressed $\beta_{2} A R$, suggesting that findings may be relevant to human breast cancer (Fig. 1b). To evaluate receptor functionality, we treated tumor cells with $\beta A R$ ligands and quantified the effect on intracellular cAMP accumulation. In each cell line, treatment with the nonselective $\beta A R$ agonist isoproterenol increased cAMP accumulation (Fig. 1c, d), consistent with previous studies [28]. This effect was blocked by the nonselective $\beta A R$ antagonist propranolol or the $\beta_{2}$ AR-selective antagonist ICI-188551. Treatment with $\beta_{1}$ AR-selective antagonist CGP-20712A or $\beta_{3}$ AR-selective antagonist L748337 did not block cAMP accumulation (Fig. 1c, d). These findings confirm that $\beta_{2} A R$ is the dominant functional $\beta A R$ in these breast cancer cell lines.

To investigate the role of $\beta A R$ signaling in invadopodia formation, LifeAct-GFP ${ }^{+}$MDA-MB-231 cells were plated on a fluorescent gelatin matrix and the effect of $\beta$-agonist isoproterenol on matrix degradation was evaluated (Fig. 2a). While actin-rich puncta were identified in the entire population, only $16 \%( \pm 4 \%)$ of tumor cells produced active invadopodia as defined by degradation of underlying matrix fluorescence (Fig. 2a, b). Treatment with isoproterenol led to an increase in the frequency of invadopodia-positive cells, with a maximum increase of 2.5 -fold in response to $0.5 \mu \mathrm{M}$ isoproterenol (Fig. 2b). In addition to increasing the frequency of invadopodia-positive cells in the population (Fig. 2b, c), isoproterenol also increased the average number of invadopodia per cell by 2.7 -fold (Fig. 2d). Treatment with the nonselective $\beta$-blocker propranolol blocked the effect 


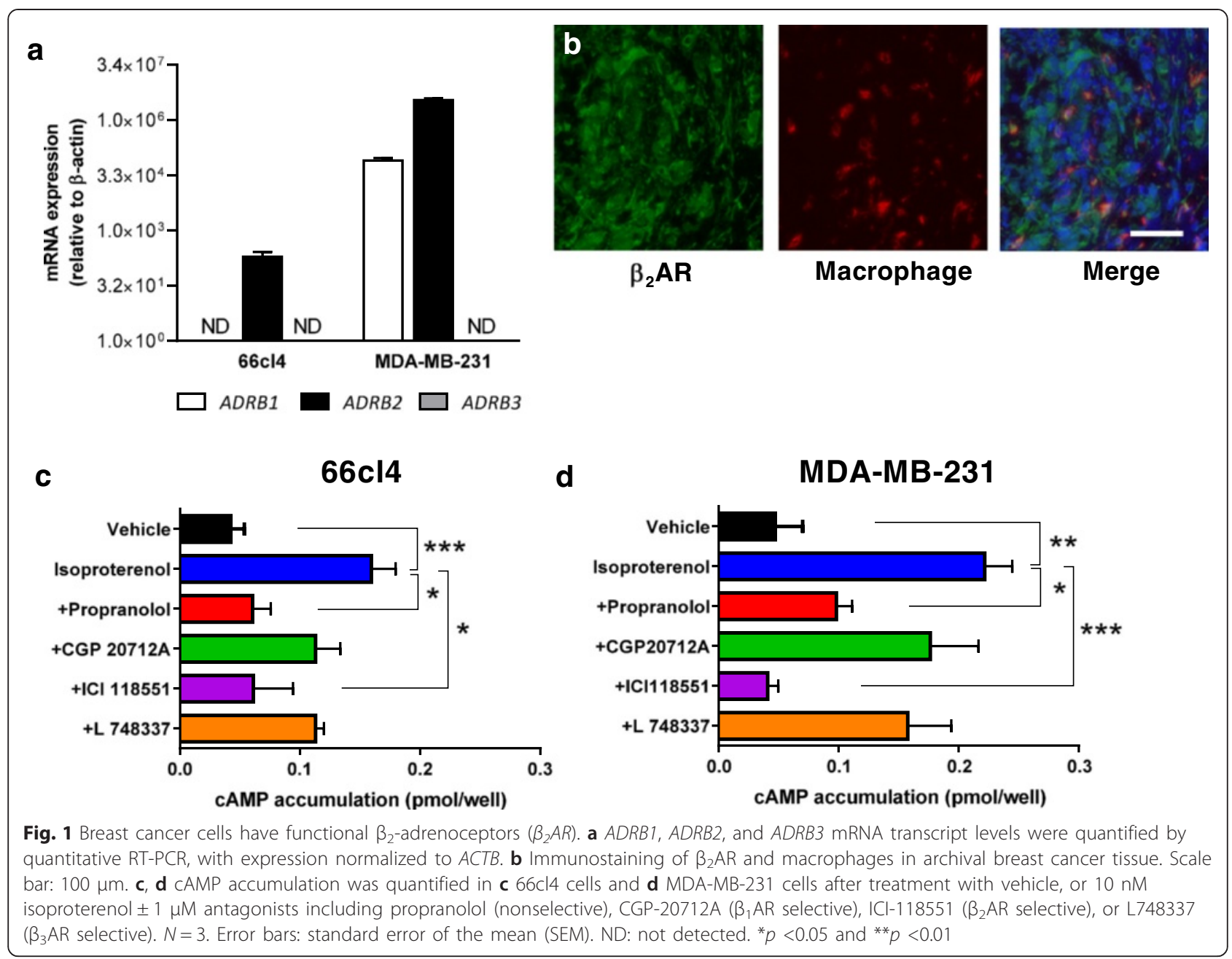

of isoproterenol on both the frequency of invadopodiapositive cells and the number of invadopodia per cell (Fig. 2c, d). This confirms that isoproterenol signals via $\beta A R$ to increase invadopodia formation. To confirm the $\beta A R$ subtype that mediates this effect, MDA-MB-231 cells were treated with selective antagonists and the effect on invadopodia was evaluated. $\beta_{2}$ AR-selective antagonist ICI-118551 blocked the increase in invadopodia formation in response to isoproterenol, whereas there was no effect of $\beta_{1}$ AR-selective antagonist CGP-20712A (Fig. 2e). To determine whether $\beta_{2} A R$ signaling is sufficient to induce invadopodia formation, cells were treated with selective $\beta A R$ agonists. $\beta_{2} A R$-selective agonist formoterol - but not $\beta_{1} \mathrm{AR}$-selective agonist xamoterol induced invadopodia formation (Fig. 2f, g), confirming a key role for $\beta_{2} A R$ signaling in the formation of invadopodia in these cells.

Formation of invadopodia requires adhesion proteins that may be sequestered from focal adhesions [6]. To investigate the effect of $\beta A R$ signaling on focal adhesions we treated breast cancer cells with isoproterenol and used immunostaining to quantify the number and length of paxillin-positive focal adhesions (Fig. 3a). Isoproterenol resulted in a concentration-dependent decrease in adhesion length and decreased number of focal adhesions per cell (Fig. 3a, b). Loss of focal adhesion-associated proteins has been linked to a decreased frequency of focal adhesions and reduced capacity for cell migration on two-dimensional surfaces [29]. Consistent with the change in focal adhesions having a functional effect on cell movement, isoproterenol decreased the migration of cancer cells on a twodimensional fibronectin surface (Fig. 3c, d).

These findings suggest that $\beta_{2} \mathrm{AR}$ signaling induces an invasive phenotype that favors tumor cell invasion in three-dimensional environments. To investigate this, MDA-MB-231 breast cancer cells were embedded in collagen matrix and cell locomotion was tracked over time. Under control conditions, cells remained rounded with little displacement from the position of origin (Fig. 4a, b). Treatment with $\beta_{2}$ AR-selective agonist formoterol induced cancer cells to elongate and form 


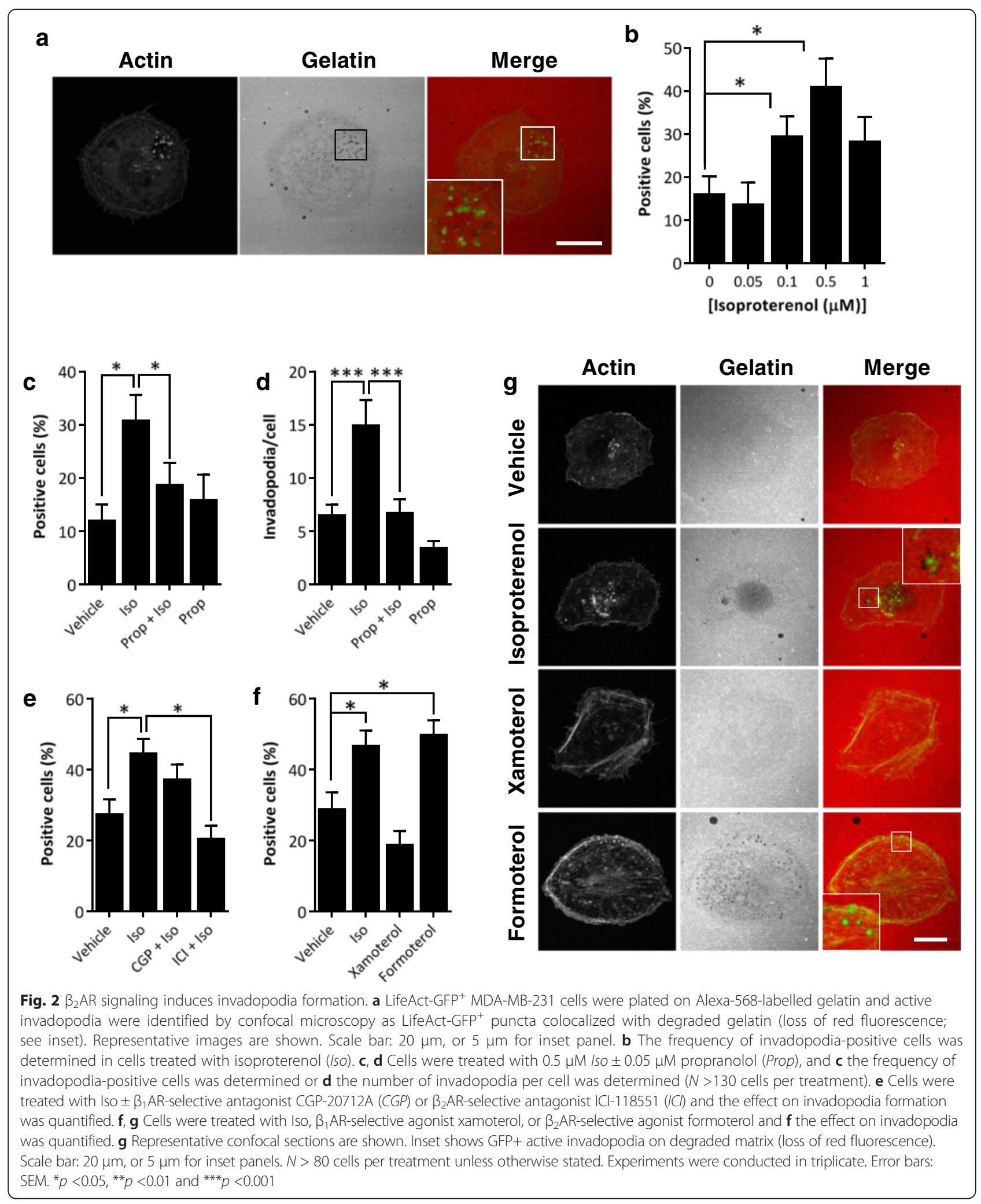

protrusions (Fig. 4a) and increased cell displacement over time (Fig. $4 \mathrm{~b}, \mathrm{c}$ ), indicating that $\beta_{2} \mathrm{AR}$ signaling is sufficient to induce invasion of MDA-MB-231 breast cancer cells. Treatment with isoproterenol similarly induced cellular protrusion formation and these effects were blocked by the $\beta_{2} \mathrm{AR}$-selective antagonist ICI- 


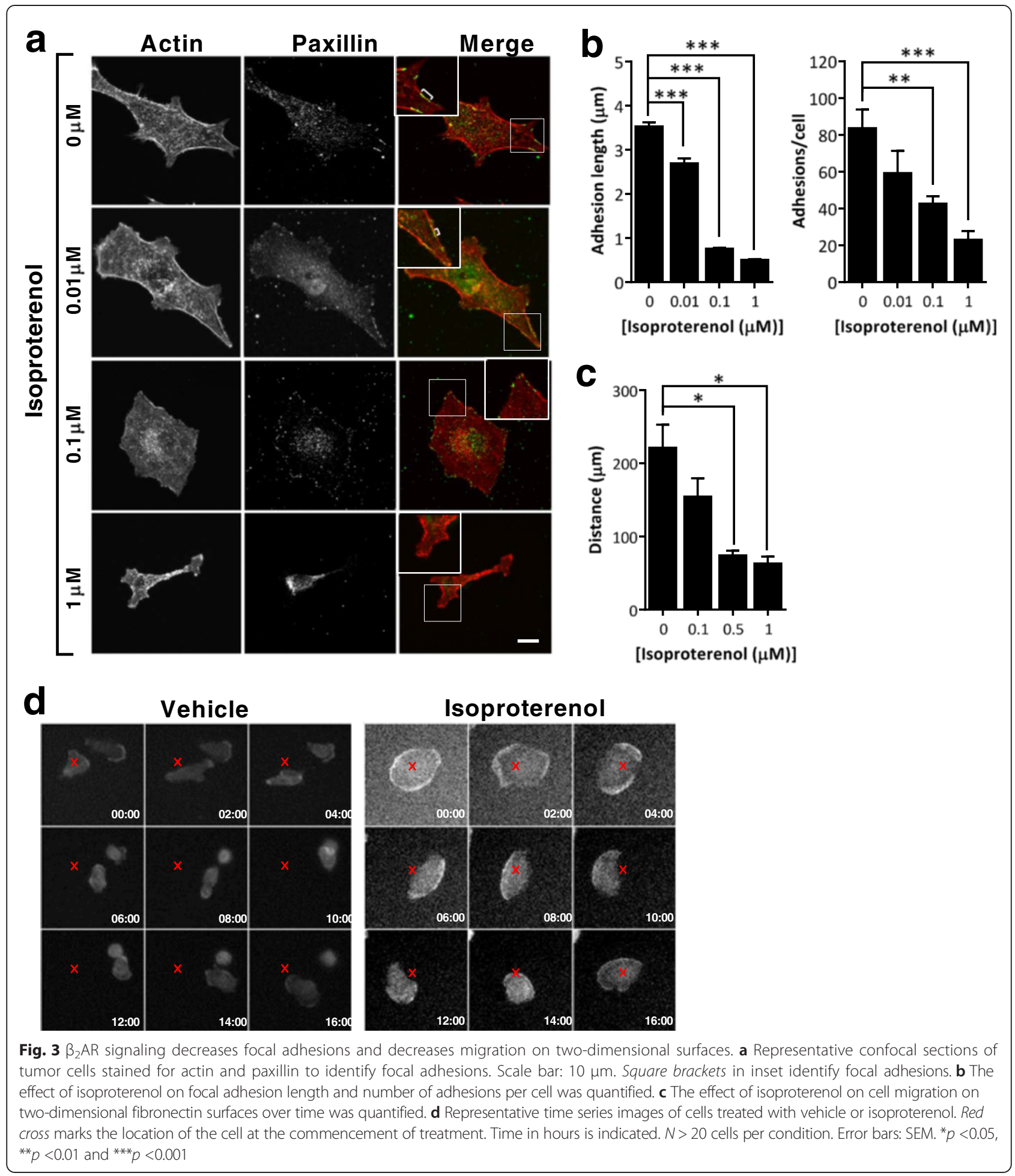

118551, confirming a role for $\beta_{2} \mathrm{AR}$ signaling in these effects on cell invasion through a three-dimensional collagen matrix. By identifying differential effects of $\beta A R$ signaling between cell movement on two-dimensional surfaces compared with migration in three-dimensional environments, these findings may reconcile seemingly inconsistent observations for the effects of endogenous neurotransmitters and $\beta A R$-selective agonists on tumor cell migration [30-33]. Because movement in threedimensional environments reflects processes that are 


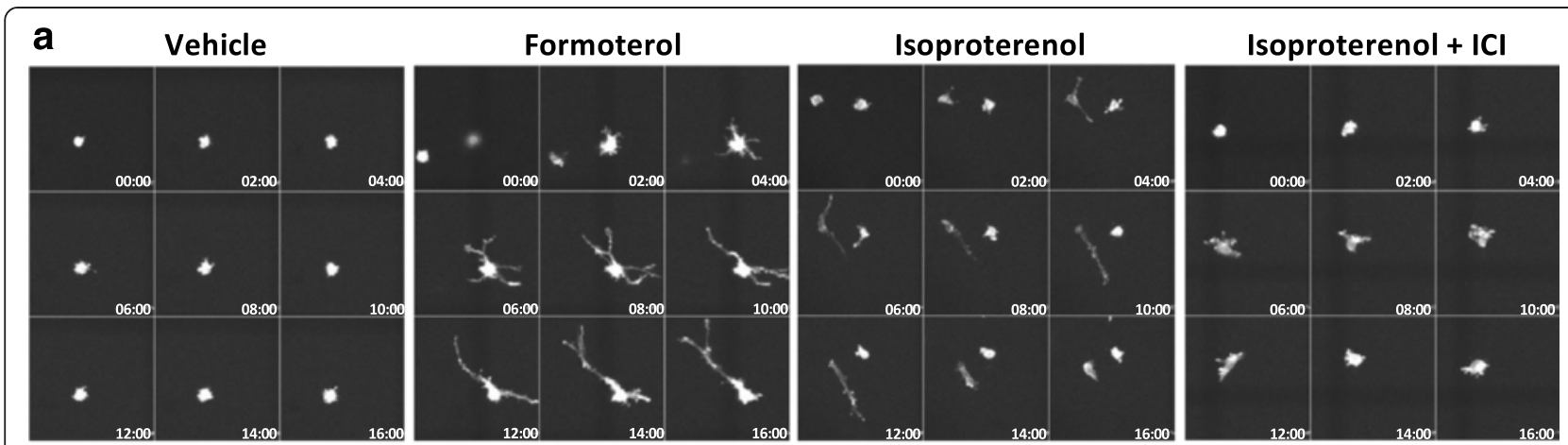

b

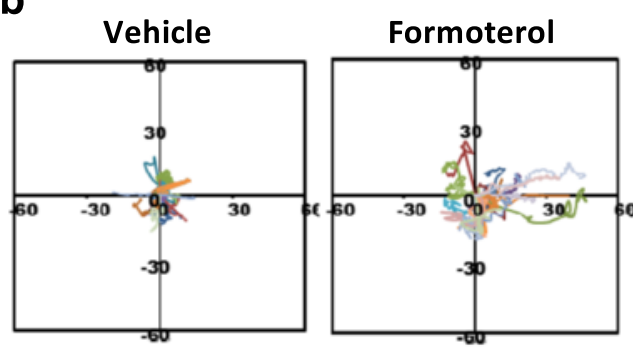

d

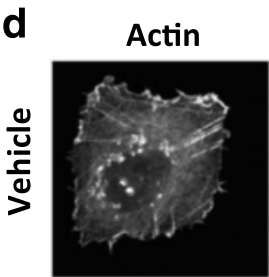

\section{autum}
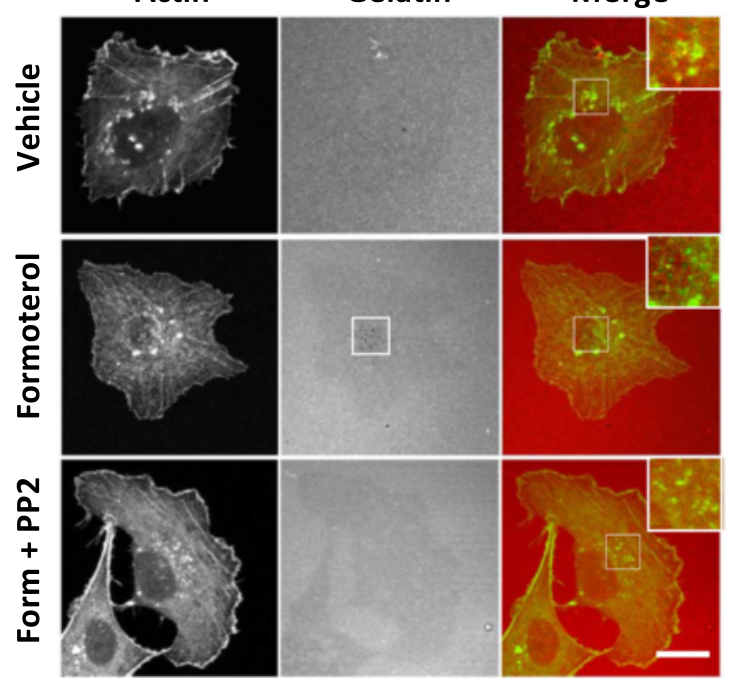

Isoproterenol

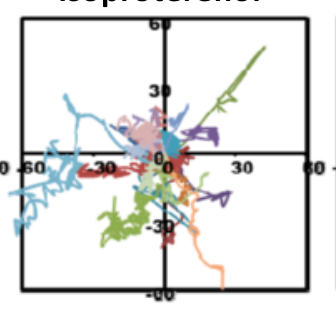

Isoproterenol + ICI

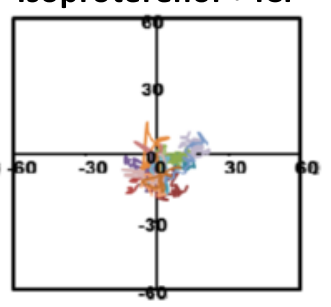

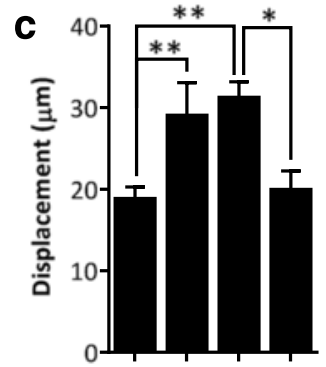

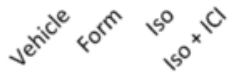

Fig. $4 \beta_{2}$ AR-induced invadopodia formation is dependent on Src and increases tumor cell invasion in a three-dimensional collagen matrix. a Representative time series images acquired by confocal microscopy showing the effect of formotorol or isoproterenol (Iso) $\pm 0.05 \mu \mathrm{M}$ ICl-118551 (ICl) on development of cell protrusions. Time in hours is indicated. b Graphical representation of track measurements is shown for 20 cells in each condition. c Quantification of cell displacement from the point of origin. d, e Representative maximum intensity projections of LifeAct-GFP MDA-MB-231 cells treated with $0.5 \mu \mathrm{M} \mathrm{PP} 2 \pm 0.5 \mu \mathrm{M}$ formoterol and examined for active invadopodia. Inset shows GFP+ green puncta (active invadopodia that have degraded underlying matrix) or yellow puncta (inactive invadopodia where GFP+ puncta have not degraded the underlying red fluorescent matrix). Scale bar: $10 \mu \mathrm{m}$, or $5 \mu \mathrm{m}$ for inset panels. e The percentage of invadopodia positive cells was quantified. $N>80$ cells per condition. $\mathbf{f}$ Graphical representation of track measurements after cells were embedded in $1 \mathrm{mg} / \mathrm{ml}$ collagen matrix and treated with $0.5 \mu \mathrm{M}$ formoterol \pm PP2 Src inhibitor. $N=20$ cells per condition. Error bars: SEM. ${ }^{*} p<0.05$ and ${ }^{* *} p<0.01$

required for tumor cell intravasation and extravasation during metastasis, these findings suggest a cellular mechanism for observations that $\beta A R$ signaling drives breast cancer progression $[12,13]$.
Src activity plays a key role in invadopodia formation $[4,6]$, and has been implicated in $\beta A R$ regulation of ovarian cancer invasion, although the cellular mechanisms are unclear [15]. To investigate the role of Src in $\beta_{2} \mathrm{AR}$ 
regulation of invadopodia, MDA-MB-231 cells were pretreated with the Src inhibitor PP2 prior to treatment with formoterol. PP2 treatment blocked the effect of $\beta_{2} \mathrm{AR}$ stimulation on the frequency of invadopodia (Fig. 4d, e), as seen by loss of matrix fluorescence. Treatment with PP2 alone did not significantly change the frequency of invadopodia compared to treatment with vehicle. Treatment with PP2 also blocked the effect of formoterol on invasion of cells in three-dimensional collagen matrix (Fig. 4f), demonstrating that $\mathrm{Src}$ is essential for $\beta_{2} \mathrm{AR}$-mediated invasion. These findings indicate that $\beta_{2} A R$ signaling regulates invadopodia formation and tumor cell invasion through canonical Src signaling pathways. $\beta A R$ activation was recently shown to phosphorylate $\operatorname{Src}$ at $\mathrm{Y}^{416}$ in addition to $S^{17}$ [15], suggesting that characterization of the activation status of Src will contribute to a more complete understanding of the molecular mechanisms involved. Additional insight may be gained from clarifying other $\beta_{2}$ AR-induced molecular changes that may amplify effects on tumor cell dissemination. For example, norepinephrine was shown to protect cancer cells from anoikis by activating and relocalizing focal adhesion kinase [34]. Coordinated regulation of invadopodia formation and reduced anoikis in response to $\beta_{2} \mathrm{AR}$-mediated neural signaling may serve to amplify the effects on tumor cell dissemination and cancer progression.

To investigate the impact of $\beta_{2} \mathrm{AR}$ regulation of tumor cell invasion in vivo, bioluminescence imaging was used to track the effect of $\beta_{2}$ AR-selective agonist formoterol on metastatic progression in an orthotopic xenograft model of breast cancer. Mice were implanted with luciferase-tagged MDA-MB-231 cells into the left fourth mammary fat pad and spontaneous metastasis was tracked by bioluminescence imaging using 1-second exposure to detect signals from primary tumors and 60second exposure to detect signals from metastases (Fig. 5a). Treatment with formoterol during tumor development accelerated the formation of metastasis (Fig. 5b). To examine whether increased metastasis was linked to increased tumor cell invasion, a subset of mammary tumors were surgically resected 9 days after tumor cell inoculation, before the onset of metastasis,
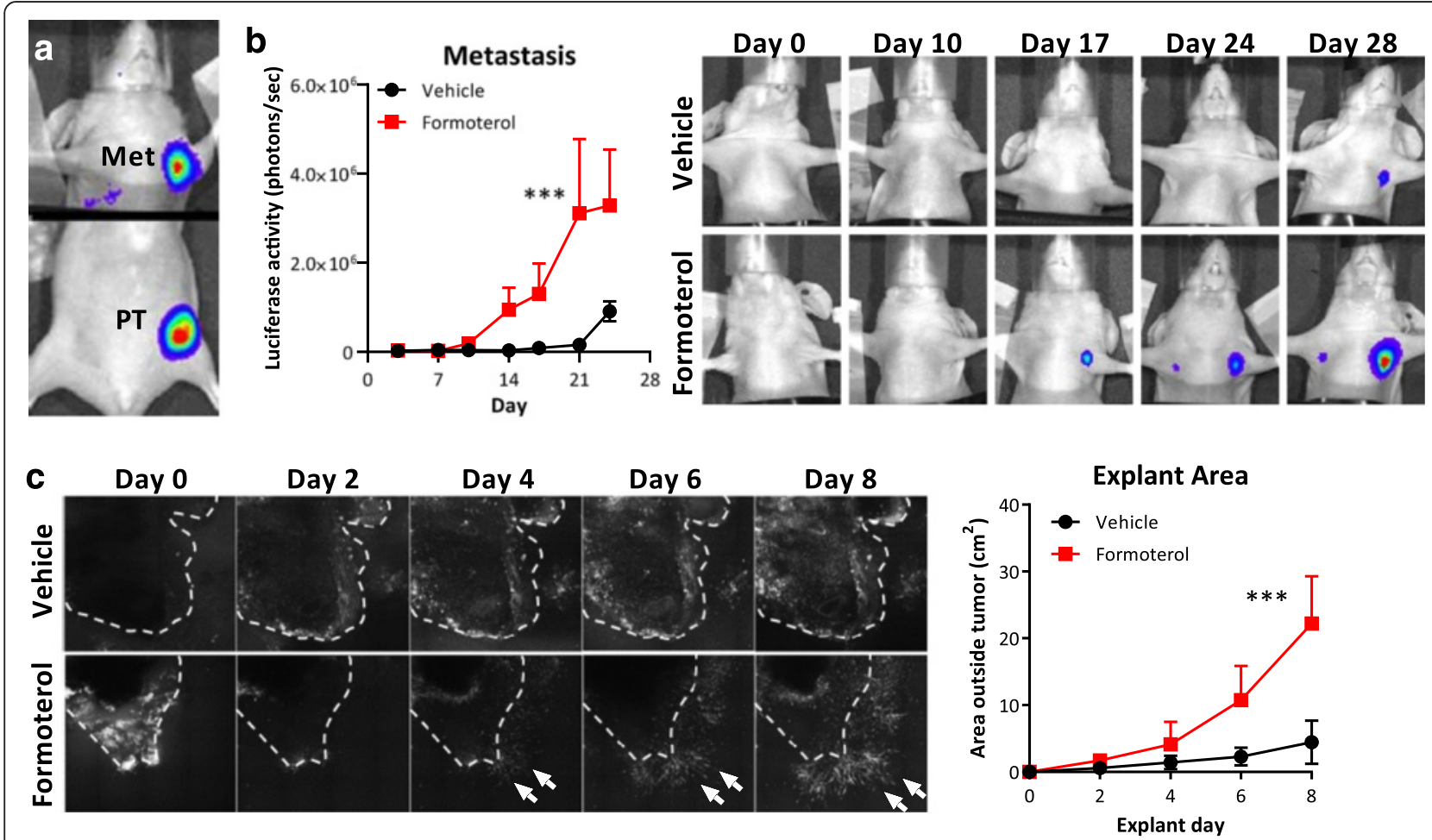

Fig. $5 \beta_{2}$ AR signaling induces tumor cell invasion and metastasis from primary mammary tumors. a Representative image of the orthotopic metastasis model. Luciferase-tagged tumor cells were injected into the fourth mammary fat pad (PT) and spontaneous metastases to the lymph node and lung (Met) were detected by optical bioluminescence imaging. Lower body exposure: 1 second. Upper body exposure: 60 seconds. Black bar separates images taken with two different exposures. b Mice were treated daily with $5 \mathrm{mg} / \mathrm{kg}$ formoterol (or saline vehicle) during tumor development and the effect of distant metastasis was quantified over time by bioluminescence imaging and expressed relative to primary tumor size. $\mathrm{N}=5$ at each time-point. c Primary tumors were resected from the mammary fat pad of vehicle vs. formoterol-treated mice at day 10 after tumor cell injection and embedded in a three-dimensional collagen matrix. Invasion of LifeAct-GFP ${ }^{+}$tumor cells beyond explant boundaries into the surrounding collagen (arrows) was imaged over 8 days by confocal microscopy and quantified. $N=5$ at each time point. Error bars: SEM. ${ }^{* * *} p<0.001$ 
and embedded in a three-dimensional collagen matrix. In contrast to tumors from vehicle-treated mice, which demonstrated minimal invasion of cells into the surrounding extracellular matrix over the 8-day imaging period, tumors from mice treated with formoterol had rapid invasion of tumor cells into the surrounding matrix (Fig. 5c). This shows that $\beta_{2} \mathrm{AR}$ signaling increases dissemination from explanted tumors, suggesting a possible mechanism for increased distant metastasis.

While recent studies of $\beta$ AR-mediated stress biology on cancer progression have focused on the impact of neural signaling on stromal cells in the tumor microenvironment $[12,14,35,36]$, the findings presented here demonstrate that tumor cells are directly responsive to $\beta A R$ signaling. By driving a shift away from focal adhesions to invadopodia formation, $\beta_{2} \mathrm{AR}$ signaling would favor tumor cells with enhanced capacity for invasion through the three-dimensional extracellular matrix of the tumor microenvironment. Metastasis is a highly inefficient process and requires successful completion of a series of inter-connected steps including dissemination from the primary tumor, survival in circulation, extravasation, and colonization at distant sites [37-39]. Failure at any stage of metastasis will result in failure of the entire process [40]. By increasing both the density of invadopodia per cell and the frequency of invadopodiapositive cells in the tumor cell population, $\beta_{2} \mathrm{AR}$ signaling may promote metastasis by enhancing the invasive capacity of cells in the tumor.

These findings suggest that blocking tumor cell responsiveness to stress signaling may protect against tumor cell invasion and metastasis. Specifically, the effects of $\beta_{2}$ AR signaling on invadopodia formation were reversed by $\beta$-blockade, providing a cellular mechanism for clinical observations that pharmacological $\beta$-blockade was linked to improved cancer outcomes [41-43]. Retrospective epidemiological studies found that $\beta$-blocker treatment of comorbid hypertension was associated with reduced metastasis and improved survival in different cancer types including breast and prostate cancers and melanoma (reviewed in [44]). The current findings show that $\beta$-blockers act directly on cancer cells to decrease their invasiveness. While not excluding that $\beta$-blockade regulates cancer progression through effects on the tumor microenvironment (e.g., modulating recruitment of inflammatory cells or angiogenesis) $[12,35]$, these findings suggest that $\beta$-blockers may be optimally targeted to patients with high tumor cell expression of $\beta_{2} \mathrm{AR}$. Recent clinical practice has preferred the use of $\beta_{1}$-selective blockers for treatment of hypertension, to avoid adverse effects on bronchoconstriction [45]. The findings presented here suggest that use of nonselective $\beta$-blockers such as propranolol may be required to favorably impact cancer outcomes. Additionally these findings may warrant the development of novel $\beta_{2}$-selective blockers that may be targeted to tumor cells to avoid adverse side effects. Finally, these findings suggest that it may be important to consider the effect of clinically used $\beta_{2} \mathrm{AR}$ agonists (e.g., by asthmatics for bronchodilation) on cancer progression.

\section{Conclusions}

Here, we present evidence that $\beta_{2} \mathrm{AR}$ signaling drives a switch from focal adhesions to invadopodia formation in breast cancer cells to increase cell invasion in threedimensional environments. These findings suggest that $\beta_{2} \mathrm{AR}$ may be a key receptor for transmission of neural signals from the tumor microenvironment to regulate behavior of tumor cells. The findings provide a plausible mechanism for accumulating evidence that chronic stress promotes cancer progression and metastasis $[12,35,46,47]$, and suggest that selective pharmacological blockade of $\beta_{2} \mathrm{AR}$ signaling pathways may be important to slow cancer progression.

\section{Abbreviations}

BAR: $\beta$-adrenoceptor; DMEM: Dulbecco's modified Eagle's medium; FBS: Fetal bovine serum; GFP: Green fluorescent protein; Iso: Isoproterenol; MMP: Matrix metalloprotease; SEM: Standard error of the mean.

\section{Competing interests}

The authors declare that they have no competing interests.

\section{Authors' contributions}

SJC conceived and designed the study, performed experiments, analyzed data, and wrote the manuscript. CPL performed experiments, analyzed data, and wrote the manuscript. $\mathrm{MH}$ performed migration experiments, analyzed data, and revised the manuscript. CKP performed CAMP experiments, analyzed data, and revised the manuscript. SA performed experiments, analyzed data, and revised the manuscript. KTC generated constructs and revised the manuscript. MEB developed software for invadopodia analysis. $\mathrm{ZH}$ analyzed data and revised the manuscript. JEB generated and provided reagents and revised the manuscript. JRL generated and provided reagents and revised the manuscript. MLH analyzed data and revised the manuscript. DF developed analysis techniques, analyzed data, and revised the manuscript. CJN developed imaging analysis software, analyzed data, and revised the manuscript. EKS conceived and designed the study, performed experiments, analyzed data, and wrote the manuscript. All authors read and approved the final manuscript.

\section{Acknowledgements}

The authors thank Prof. Stephen Hill and Prof. Frederic Hollande for thoughtful discussion of this research and critically reviewing the manuscript, and the Monash Institute of Pharmaceutical Sciences Imaging, Flow Cytometry and Analysis Core for imaging and data analysis support. This research was funded by the Australian National Health and Medical Research Council (1008865), the Australian Research Council (LE110100125), the National Cancer Institute (CA160890), the National Breast Cancer Foundation (ECR-11-11) to EKS, a NHMRC RD Wright Fellowship to MLH (1061687), and MIPS-DDB Strategic Funding.

\section{Author details}

${ }^{1}$ Drug Discovery Biology Theme, Monash Institute of Pharmaceutical Sciences, Monash University, Parkville, VIC 3052, Australia. ${ }^{2}$ Department of Cell \& Developmental Biology and Lineberger Comprehensive Cancer Center, School of Medicine, The University of North Carolina Chapel Hill, Chapel Hill, NC 27599, USA. ${ }^{3}$ Department of Biomedical Engineering, Duke University, Durham, NC 27708, USA. ${ }^{4}$ Department of Mathematics and Statistics, The 
University of Melbourne, Parkville, VIC 3010, Australia. ${ }^{5}$ Cousins Center for PNI, UCLA Semel Institute, and Jonsson Comprehensive Cancer Center, University of California Los Angeles, Los Angeles, CA 90095, USA. ${ }^{6}$ Division of Cancer Surgery, Peter MacCallum Cancer Centre, East Melbourne, VIC 3002, Australia. ${ }^{7}$ Current address: Peter MacCallum Cancer Centre, East Melbourne, VIC 3002, Australia

\section{Received: 7 July 2015 Accepted: 9 November 2015} Published online: 25 November 2015

\section{References}

1. Weigelt B, Peterse JL, Van't Veer LJ. Breast cancer metastasis: markers and models. Nat Rev Cancer. 2005;5(8):591-602.

2. Baldassarre M, Ayala I, Beznoussenko G, Giacchetti G, Machesky LM, Luini A, et al. Actin dynamics at sites of extracellular matrix degradation. Eur J Cell Biol. 2006;85(12):1217-31.

3. Chen WT, Chen JM, Parsons SJ, Parsons JT. Local degradation of fibronectin at sites of expression of the transforming gene product pp60src. Nature. 1985:316(6024):156-8.

4. Chen WT. Proteolytic activity of specialized surface protrusions formed at rosette contact sites of transformed cells. J Exp Zool. 1989;251(2): 167-85.

5. Brabek J, Constancio SS, Shin NY, Pozzi A, Weaver AM, Hanks SK. CAS promotes invasiveness of Src-transformed cells. Oncogene. 2004;23(44): 7406-15.

6. Chan $\mathrm{KT}$, Cortesio $\mathrm{CL}$, Huttenlocher A. FAK alters invadopodia and focal adhesion composition and dynamics to regulate breast cancer invasion. The Journal of cell biology. 2009;185(2):357-70.

7. Quail DF, Joyce JA. Microenvironmental regulation of tumor progression and metastasis. Nat Med. 2013;19(11):1423-37.

8. Shi M, Liu D, Duan H, Qian L, Wang L, Niu L, et al. The beta2-adrenergic receptor and Her2 comprise a positive feedback loop in human breast cancer cells. Breast Cancer Res Treat. 2011;125(2):351-62.

9. Powe DG, Voss MJ, Habashy HO, Zanker KS, Green AR, Ellis IO, et al. Alphaand beta-adrenergic receptor (AR) protein expression is associated with poor clinical outcome in breast cancer: an immunohistochemical study. Breast Cancer Res Treat. 2011:130(2):457-63.

10. Kobilka B. Adrenergic receptors as models for $\mathrm{G}$ protein-coupled receptors. Annu Rev Neurosci. 1992:15:87-114.

11. Landen Jr CN, Lin YG, Armaiz Pena GN, Das PD, Arevalo JM, Kamat AA, et al. Neuroendocrine modulation of signal transducer and activator of transcription-3 in ovarian cancer. Cancer Res. 2007;67(21):10389-96.

12. Sloan EK, Priceman SJ, Cox BF, Yu S, Pimentel MA, Tangkanangnukul V, et al. The sympathetic nervous system induces a metastatic switch in primary breast cancer. Cancer research. 2010;70(18):7042-52.

13. Lamkin DM, Sung HY, Yang GS, David JM, Ma JC, Cole SW, et al. alpha2-Adrenergic blockade mimics the enhancing effect of chronic stress on breast cancer progression. Psychoneuroendocrinology. 2015;51: 262-70

14. Kim-Fuchs C, Le CP, Pimentel MA, Shackleford D, Ferrari D, Angst E, et al. Chronic stress accelerates pancreatic cancer growth and invasion: a critical role for beta-adrenergic signaling in the pancreatic microenvironment. Brain, behavior, and immunity. 2014:40:40-7.

15. Armaiz-Pena GN, Allen JK, Cruz A, Stone RL, Nick AM, Lin YG, et al. Src activation by beta-adrenoreceptors is a key switch for tumour metastasis. Nature Communications. 2013:4:1403.

16. Du J, Li L, Ou Z, Kong C, Zhang Y, Dong Z, et al. FOXC1, a target of polycomb, inhibits metastasis of breast cancer cells. Breast Cancer Res Treat. 2012;131(1):65-73

17. Kaminskas LM, Ascher DB, McLeod VM, Herold MJ, Le CP, Sloan EK, et al. PEGylation of interferon alpha2 improves lymphatic exposure after subcutaneous and intravenous administration and improves antitumour efficacy against lymphatic breast cancer metastases. J Control Release. 2013; 168(2):200-8.

18. Eckhardt BL, Parker BS, van Laar RK, Restall CM, Natoli AL, Tavaria MD, et al. Genomic analysis of a spontaneous model of breast cancer metastasis to bone reveals a role for the extracellular matrix. Mol Cancer Res. 2005:3(1):1-13.

19. Berginski ME, Creed SJ, Cochran S, Roadcap DW, Bear JE, Gomez SM. Automated analysis of invadopodia dynamics in live cells. PeerJ. 2014;2: e462.
20. Kim JH, Lee SR, Li LH, Park HJ, Park JH, Lee KY, et al. High cleavage efficiency of a 2A peptide derived from porcine teschovirus-1 in human cell lines, zebrafish and mice. PLoS One. 2011;6(4):e18556

21. Cai L, Marshall TW, Uetrecht AC, Schafer DA, Bear JE. Coronin 1B coordinates Arp2/3 complex and cofilin activities at the leading edge. Cell. 2007;128(5): 915-29.

22. Dobson AJ, Barnett AG. An Introduction to Generalized Linear Models. CRC Press; 2008.

23. Hsu J. Multiple Comparisons: Theory and Methods. CRC Press; 1996.

24. Becker RA, Chambers AM, Wilks AR. The new S language: a programming environment for data analysis and graphics Wadsworth and Brooks/Cole Advanced Books \& Software Monterey. USA: CA; 1988.

25. Bretz F, Hothorn T, Westfall P. Multiple Comparisons using R. CRC Press; 2010.

26. Kolak M, Yki-Jarvinen $H$, Kannisto K, Tiikkainen M, Hamsten A, Eriksson P, et al. Effects of chronic rosiglitazone therapy on gene expression in human adipose tissue in vivo in patients with type 2 diabetes. J Clin Endocrinol Metab. 2007;92(2):720-4.

27. Jung KM, Clapper JR, Fu J, D'Agostino G, Guijarro A, Thongkham D, et al 2-arachidonoylglycerol signaling in forebrain regulates systemic energy metabolism. Cell Metab. 2012;15(3):299-310.

28. Slotkin TA, Zhang J, Dancel R, Garcia SJ, Willis C, Seidler FJ. Betaadrenoceptor signaling and its control of cell replication in MDA-MB231 human breast cancer cells. Breast Cancer Res Treat. 2000;60(2): 153-66.

29. Ren XD, Kiosses WB, Sieg DJ, Otey CA, Schlaepfer DD, Schwartz MA. Focal adhesion kinase suppresses Rho activity to promote focal adhesion turnover. J Cell Sci. 2000;113(Pt 20):3673-8.

30. Yamanaka Y, Mammoto T, Kirita T, Mukai M, Mashimo T, Sugimura M, et al: Epinephrine inhibits invasion of oral squamous carcinoma cells by modulating intracellular CAMP. Cancer Lett. 2002;176(2):143-8.

31. Pullar CE, Le Provost GS, O'Leary AP, Evans SE, Baier BS. Isseroff RR: beta2AR antagonists and beta2AR gene deletion both promote skin wound repair processes. J Invest Dermatol. 2012;132(8):2076-84.

32. Iseri OD, Sahin Fl, Terzi YK, Yurtcu E, Erdem SR, Sarialioglu F. betaAdrenoreceptor antagonists reduce cancer cell proliferation, invasion, and migration. Pharm Biol 2014:1-8.

33. Stock AM, Powe DG, Hahn SA, Troost G, Niggemann B, Zanker KS, et al. Norepinephrine inhibits the migratory activity of pancreatic cancer cells. Exp Cell Res. 2013;319(12):1744-58.

34. Sood AK, Armaiz-Pena GN, Halder J, Nick AM, Stone RL, Hu W, et al. Adrenergic modulation of focal adhesion kinase protects human ovarian cancer cells from anoikis. J Clin Invest. 2010;120(5):1515-23.

35. Thaker PH, Han LY, Kamat AA, Arevalo JM, Takahashi R, Lu C, et al. Chronic stress promotes tumor growth and angiogenesis in a mouse model of ovarian carcinoma. Nature medicine. 2006;12(8):939-44.

36. Lamkin DM, Sloan EK, Patel AJ, Chiang BS, Pimentel MA, Ma JC, et al. Chronic stress enhances progression of acute lymphoblastic leukemia via beta-adrenergic signaling. Brain, behavior, and immunity. 2012;26(4): 635-41.

37. Wyckoff JB, Jones JG, Condeelis JS, Segall JE. A critical step in metastasis: in vivo analysis of intravasation at the primary tumor. Cancer Res. 2000;60(9): 2504-11.

38. Fidler IJ. Metastasis: guantitative analysis of distribution and fate of tumor embolilabeled with 125 I-5-iodo-2'-deoxyuridine. J Natl Cancer Inst. 1970; 45(4):773-82.

39. Chiang AC, Massague J. Molecular basis of metastasis. N Engl J Med. 2008; 359(26):2814-23.

40. Chambers AF, Naumov GN, Varghese HJ, Nadkarni KV, MacDonald IC, Groom AC. Critical steps in hematogenous metastasis: an overview. Surg Oncol Clin N Am. 2001;10(2):243-55. vii.

41. Melhem-Bertrandt A, Chavez-Macgregor M, Lei X, Brown EN, Lee RT, MericBernstam F, et al. Beta-blocker use is associated with improved relapse-free survival in patients with triple-negative breast cancer. J Clin Oncol. 2011; 29(19):2645-52.

42. Barron TI, Connolly RM, Sharp L, Bennett K, Visvanathan K. Beta blockers and breast cancer mortality: a population- based study. J Clin Oncol. 2011;29(19): 2635-44.

43. Botteri E, Munzone E, Rotmensz N, Cipolla C, De Giorgi V, Santillo B, et al. Therapeutic effect of beta-blockers in triple-negative breast cancer postmenopausal women. Breast Cancer Res Treat. 2013;140(3):567-75. 
44. Le CP, Karnezis T, Achen MG, Stacker SA, Sloan EK. Lymphovascular and neural regulation of metastasis: shared tumour signalling pathways and novel therapeutic approaches. Best practice \& research Clinical anaesthesiology. 2013;27(4):409-25.

45. Arcangelo V, Peterson A. Pharmacotherapeutics for Advanced Practice: A Practical Approach. Lippincott Williams and Wilkins; 2006.

46. Chida Y, Hamer M, Wardle J, Steptoe A. Do stress-related psychosocial factors contribute to cancer incidence and survival? Nat Clin Pract Oncol. 2008.

47. Hassan S, Karpova Y, Baiz D, Yancey D, Pullikuth A, Flores A, et al. Behavioral stress accelerates prostate cancer development in mice. J Clin Invest. 2013; 123(2):874-86.

\section{Submit your next manuscript to BioMed Central and take full advantage of:}

- Convenient online submission

- Thorough peer review

- No space constraints or color figure charges

- Immediate publication on acceptance

- Inclusion in PubMed, CAS, Scopus and Google Scholar

- Research which is freely available for redistribution 